\title{
Orthospectra of geodesic laminations and dilogarithm identities on moduli space
}

\author{
MARTIN BRIDGEMAN
}

\begin{abstract}
Given a measured lamination $\lambda$ on a finite area hyperbolic surface we consider a natural measure $M_{\lambda}$ on the real line obtained by taking the push-forward of the volume measure of the unit tangent bundle of the surface under an intersection function associated with the lamination. We show that the measure $M_{\lambda}$ gives summation identities for the Rogers dilogarithm function on the moduli space of a surface.
\end{abstract}

$32 \mathrm{G} 15 ; 11 \mathrm{M} 36$

\section{Introduction}

Let $S$ be a closed hyperbolic surface and $\lambda$ a geodesic lamination on $S$. We let $\Omega$ be the volume measure on the unit tangent bundle $T_{1}(S)$. We let $\alpha(v)$ be the longest geodesic arc containing $v$ as a tangent vector and which does not intersect $\lambda$ transversely in its interior. Generically $\alpha(v)$ will be a geodesic arc with endpoints on $\lambda$.

We define the function $L: T_{1}(S) \rightarrow \overline{\mathbb{R}}$ by letting $L(v)=$ Length $(\alpha(v))$. We note that $L(v)$ is measurable but can be infinite. We define measure $M_{\lambda}$ on the real line by $M_{\lambda}=L_{*} \Omega$. Then $M_{\lambda}$ is a measure describing the distribution of the lengths of $\alpha(v)$.

We cut $S$ along $\lambda$ to obtain a surface with boundary denoted $S_{\lambda}$. A $\lambda$-cusp of $S$ is an ideal vertex of a component of $S_{\lambda}$. We let $N_{\lambda}$ be the number of $\lambda$-cusps of $S$. We denote by $\left\{\alpha_{i}\right\}$ the geodesic arcs in $S_{\lambda}$ which have endpoints perpendicular to $\partial S_{\lambda} \subseteq \lambda$ and denote the length of $\alpha_{i}$ by $l_{i}$. We note that if a component of $S_{\lambda}$ is an ideal $k$-gon then there are a finite number of geodesics $\alpha_{i}$ in this component. Otherwise there are an infinite number. We call the set $\left\{l_{i}\right\}$ (with multiplicities) the $\lambda$-orthospectrum. By doubling $S-\lambda$ we see that the $\lambda$-orthospectrum corresponds to a subset of the closed geodesics of a finite area surface and therefore is a countable set. We prove the following length spectrum identity

$$
\sum_{i} \mathcal{L}\left(\frac{1}{\cosh ^{2} \frac{l_{i}}{2}}\right)=\frac{\pi^{2}}{12}\left(6|\chi(S)|-N_{\lambda}\right)
$$

where $\mathcal{L}$ is a Rogers dilogarithm function (described below). 


\subsection{Orthospectra identities}

The orthospectrum was introduced by Basmajian [1] in studying hyperbolic $n$-manifolds $M$ with totally geodesic boundaries. In the paper Basmajian shows that the volume of the boundary is given by the following orthospectrum identity

$$
\operatorname{Vol}(\partial M)=\sum_{l \in \Lambda_{M}} V_{n-1}(\log \operatorname{coth}(l / 2))
$$

where $\Lambda(M)$ is the orthospectrum of $M$, and $V_{n}(r)$ is the volume of a hyperbolic ball of radius $r$ in $\mathbb{H}^{n}$.

In an earlier paper [4], the author and Kahn generalize the length spectrum identity (1) above to the case of a hyperbolic $n$-manifold with geodesic boundary to obtain an identity giving the volume of the manifold in terms of the orthospectrum

$$
\operatorname{Vol}(M)=\sum_{l \in \Lambda_{M}} F_{n}(l)
$$

where $F_{n}$ are explicitly described functions. Calegari [6;5] gives an alternate derivation of these length spectrum identities.

\section{Dilogarithms and polylogarithms}

The $k^{\text {th }}$ polylogarithm function $\mathrm{Li}_{k}$ is defined by the Taylor series

$$
\operatorname{Li}_{k}(z)=\sum_{i=1}^{\infty} \frac{z^{n}}{n^{k}}
$$

for $|z|<1$ and by analytic continuation to $\mathbb{C}$. In particular

$$
\operatorname{Li}_{0}(z)=\frac{1}{1-z} \quad \operatorname{Li}_{1}(z)=-\log (1-z) .
$$

Also

$$
\operatorname{Li}_{k}^{\prime}(z)=\frac{\operatorname{Li}_{k-1}(z)}{z} \quad \text { giving } \quad \operatorname{Li}_{k}(z)=\int_{0}^{z} \frac{\operatorname{Li}_{k-1}(z)}{z} d z .
$$

Also the functions $\operatorname{Li}_{k}$ are related to the Riemann $\zeta$ function by $\operatorname{Li}_{k}(1)=\zeta(k)$.

The dilogarithm function is the function $\operatorname{Li}_{2}(z)$ and is given by

$$
\operatorname{Li}_{2}(z)=-\int_{0}^{z} \frac{\log (1-z)}{z} d z
$$


Below is a brief description of some properties of the dilogarithm function. They can all be found in 1991 survey "Structural Properties of Polylogarithms" by L Lewin [9]. From the power series representation, it is easy to see that the dilogarithm function satisfies the functional equation

$$
\mathrm{Li}_{2}(z)+\mathrm{Li}_{2}(-z)=\frac{1}{2} \mathrm{Li}_{2}\left(z^{2}\right) .
$$

Other functional relations of the dilogarithm can be best described by normalizing the dilogarithm function. The (extended) Rogers $\mathcal{L}$-function [11] is defined by

$$
\mathcal{L}(x)=\mathrm{Li}_{2}(x)+\frac{1}{2} \log |x| \log (1-x) \quad x \leq 1 .
$$

In terms of the Rogers $\mathcal{L}$-function, Euler's reflection relations for the dilogarithm are:

$$
\begin{array}{cl}
\mathcal{L}(x)+\mathcal{L}(1-x)=\mathcal{L}(1)=\frac{\pi^{2}}{6} & 0 \leq x \leq 1 \\
\mathcal{L}(-x)+\mathcal{L}\left(-x^{-1}\right)=2 \mathcal{L}(-1)=-\frac{\pi^{2}}{6} & x>0
\end{array}
$$

Also in terms of $\mathcal{L}$, Landen's identity is

$$
\mathcal{L}\left(\frac{-x}{1-x}\right)=-\mathcal{L}(x) \quad 0<x<1
$$

and Abel's functional equation is

$$
\mathcal{L}(x)+\mathcal{L}(y)=\mathcal{L}(x y)+\mathcal{L}\left(\frac{x(1-y)}{1-x y}\right)+\mathcal{L}\left(\frac{y(1-x)}{1-x y}\right) .
$$

Also a closed form for $\mathcal{L}(x)$ is known for certain values of $x$ including

$$
\begin{aligned}
\mathcal{L}(1) & =\frac{\pi^{2}}{6} & \mathcal{L}\left(\frac{1}{2}\right) & =\frac{\pi^{2}}{12} \\
\mathcal{L}\left(\phi^{-1}\right) & =\frac{\pi^{2}}{10} & \mathcal{L}\left(1-\phi^{-1}\right) & =\frac{\pi^{2}}{15}
\end{aligned}
$$

where $\phi$ is the golden ratio.

Finally we note that Ramanujan found the following linear identities (see Berndt [2] and Gordon-McIntosh [7])

$$
6 \mathcal{L}\left(\frac{1}{3}\right)-\mathcal{L}\left(\frac{1}{9}\right)=\frac{\pi^{2}}{3} \quad 3 \mathcal{L}\left(\frac{1}{4}\right)+\mathcal{L}\left(\frac{1}{9}\right)=\frac{\pi^{2}}{6} .
$$




\section{Statement of results}

The main result of the paper is the following:

Main Theorem There exists a function $\rho: \mathbb{R}^{2} \rightarrow \mathbb{R}$ such that infinitesimally

$$
d M_{\lambda}=\left(\frac{4 N_{\lambda} x^{2}}{\sinh ^{2} x}+\sum_{i} \rho\left(l_{i}, x\right)\right) d x
$$

where $N_{\lambda}$ is the number of $\lambda$-cusps of $S$. Furthermore the total mass of the measure $\rho(l, x) d x$ on the real line is given by

$$
F(l)=\int_{0}^{\infty} \rho(l, x) d x=8 \mathcal{L}\left(\frac{1}{\cosh ^{2} \frac{l_{i}}{2}}\right) .
$$

In particular the measure $M_{\lambda}$ depends only on the $\lambda$-orthospectrum.

\section{The Length Spectrum Identity}

As $M_{\lambda}=L_{*} \Omega, M_{\lambda}$ has total mass equal to the volume of $T_{1}(S)$. Therefore $M_{\lambda}(\mathbb{R})=$ $\Omega\left(T_{1}(S)\right)=4 \pi^{2}|\chi(S)|$. Summing up the masses of measures in the Main Theorem we immediately obtain the following.

Length Spectrum Identity Theorem Let $\lambda$ be a geodesic lamination on a finite area hyperbolic surface $S$. Then the $\lambda$-orthospectrum satisfies

$$
\sum_{i} \mathcal{L}\left(\frac{1}{\cosh ^{2} \frac{l_{i}}{2}}\right)=\frac{\pi^{2}\left(6|\chi(S)|-N_{\lambda}\right)}{12}
$$

or, equivalently,

$$
\sum_{i} \mathcal{L}\left(-\frac{1}{\sinh ^{2} \frac{l_{i}}{2}}\right)=\frac{\pi^{2}\left(6 \chi(S)+N_{\lambda}\right)}{12}
$$

By Landen's identity (see equation (3)) we have

$$
\mathcal{L}\left(\frac{1}{\cosh ^{2}\left(\frac{l}{2}\right)}\right)=-\mathcal{L}\left(-\frac{1}{\sinh ^{2}\left(\frac{l}{2}\right)}\right) .
$$

Thus we can see that the second form of the Length Spectrum Identity corresponds to the first via Landen's identity. 


\section{The Length Spectrum Identity on moduli space}

We note that if $S$ is a connected hyperbolic surface of finite area with non-empty geodesic boundary, letting $\lambda=\partial S$ then the Length Spectrum Identity gives a summation identity on the Moduli space $\operatorname{Mod}(S)$ of $S$. In this case the Euler characteristic $\chi(S)$ can be a fraction and is defined such that $2 \pi \chi(S)$ is the negative of the area of $S$. This relation is an infinite relation except in the case when $S$ is an ideal polygon. In this case we will show that these finite identities include the classical dilogarithm identities described above.

\subsection{Classical identities and the moduli space of ideal polygons}

For $S$ an ideal n-gon, the Length Spectrum Identity is a finite summation relation. We will show that the associated relations give an infinite list of finite relations including the classical identities stated in the previous section.

If $\left\{l_{i}\right\}$ is a $\lambda$-orthospectrum, we will define two parameterizations by letting

$$
a_{i}=-\frac{1}{\sinh ^{2} \frac{l_{i}}{2}} \quad b_{i}=\frac{1}{\cosh ^{2} \frac{l_{i}}{2}} .
$$

We now consider the Poincare disk model and let $x_{i}, i=1, \ldots, n$ be the vertices in anticlockwise cyclic ordering around the circle. Let $s_{i}$ be the side $x_{i} x_{i+i}$. Let $l_{i j}$ be the length of the diagonal between $s_{i}$ and $s_{j}$ for $|i-j| \geq 2$. We define the cross-ratio by

$$
\left[z_{1}, z_{2}, z_{3}, z_{4}\right]=\frac{\left(z_{1}-z_{2}\right)\left(z_{4}-z_{3}\right)}{\left(z_{1}-z_{3}\right)\left(z_{4}-z_{2}\right)} .
$$

As the cross ratio is invariant under Möbius transformations, we map the quadruple $\left(x_{i}, x_{i+1}, x_{j}, x_{j+1}\right)$ to $\left(-1,1, e^{l_{i j}},-e^{l_{i j}}\right)$. Then

$$
\begin{aligned}
& {\left[x_{i}, x_{i+1}, x_{j}, x_{j+1}\right]=\left[-1,1, e^{l_{i j}},-e^{l_{i j}}\right]=} \\
& \quad-\frac{(-1-1)\left(-e^{l_{i j}}-e^{l_{i j}}\right)}{\left(-1-e^{l_{i j}}\right)\left(-e^{l_{i j}}-1\right)}=\frac{4 e^{l_{i j}}}{\left(e^{l_{i j}}+1\right)^{2}}=\frac{1}{\cosh ^{2}\left(\frac{l_{i j}}{2}\right)} .
\end{aligned}
$$

As $S$ has area $(n-2) \pi$ and $n$ cusps, $\chi(S)=(n-2) / 2$ and $N_{\lambda}=n$. Thus the Length Spectrum Identity becomes

$$
\sum_{i, j} \mathcal{L}\left(\left[x_{i}, x_{i+1}, x_{j}, x_{j+1}\right]\right)=\frac{(n-3) \pi^{2}}{6}
$$


where the sum is over all ordered pairs $i, j$ such that the sides $s_{i}, s_{j}$ are disjoint (at infinity). In terms of dilogarithms we get

$$
\begin{aligned}
& \sum_{i, j} \operatorname{Li}_{2}\left(\left[x_{i}, x_{i+1}, x_{j}, x_{j+1}\right]\right)= \\
& \quad \frac{(n-3) \pi^{2}}{6}-\frac{1}{2} \sum_{i, j} \log \left(1-\left[x_{i}, x_{i+1}, x_{j}, x_{j+1}\right]\right) \log \left(\left[x_{i}, x_{i+1}, x_{j}, x_{j+1}\right]\right) .
\end{aligned}
$$

\subsection{Some cases}

Quadrilateral The ideal quadrilateral has 4 cusps and two ortholengths $l_{1}, l_{2}$. By elementary hyperbolic geometry we have $\sinh \left(l_{1} / 2\right) \cdot \sinh \left(l_{2} / 2\right)=1$. Therefore $a_{1} \cdot a_{2}=1$ and letting $a=a_{1}$ the Length Spectrum Identity is equivalent to the the classical reflection identity of Euler.

$$
\mathcal{L}(a)+\mathcal{L}\left(a^{-1}\right)=-\frac{\pi^{2}}{6}
$$

Also we have

$$
\begin{aligned}
& b_{2}=\frac{1}{\cosh ^{2}\left(l_{2} / 2\right)}=\frac{1}{1+\sinh ^{2}\left(l_{2} / 2\right)}= \frac{1}{1+\frac{1}{\sinh ^{2}\left(l_{1} / 2\right)}}= \\
& \frac{\sinh ^{2}\left(l_{1} / 2\right)}{\cosh ^{2}\left(l_{1} / 2\right)}=1-\frac{1}{\cosh ^{2}\left(l_{1} / 2\right)}=1-b_{1} .
\end{aligned}
$$

Thus letting $b=b_{1}$, the Length Spectrum identity is equivalent to the Euler reflection identity

$$
\mathcal{L}(b)+\mathcal{L}(1-b)=\frac{\pi^{2}}{6}
$$

Pentagon and Abel's Identity If we choose a general ideal pentagon then there are 5 diagonals and therefore 5 parameters $a_{i}$. We send three of the vertices to $0,1, \infty$ and the other two to $u, v$ with $0<u<v<1$. Then the cross ratios in terms of $u, v$ are

$$
u, \quad 1-v, \quad \frac{v-u}{v}, \quad \frac{v-u}{1-u}, \quad \frac{u(1-v)}{v(1-u)} .
$$

Putting into the equation we obtain the following equation:

$$
\mathcal{L}(u)+\mathcal{L}(1-v)+\mathcal{L}\left(\frac{v-u}{v}\right)+\mathcal{L}\left(\frac{v-u}{1-u}\right)+\mathcal{L}\left(\frac{u(1-v)}{v(1-u)}\right)=\frac{\pi^{2}}{3}
$$


Letting $x=u / v, y=v$, then we get

$$
\mathcal{L}(x y)+\mathcal{L}(1-y)+\mathcal{L}(1-x)+\mathcal{L}\left(\frac{y(1-x)}{1-x y}\right)+\mathcal{L}\left(\frac{x(1-y)}{1-x y}\right)=\frac{\pi^{2}}{3} .
$$

Now by applying Euler's reflection identities for $x, y$, we obtain Abel's identity for the Rogers $\mathcal{L}$-function:

$$
\mathcal{L}(x)+\mathcal{L}(y)=\mathcal{L}(x y)+\mathcal{L}\left(\frac{y(1-x)}{1-x y}\right)+\mathcal{L}\left(\frac{x(1-y)}{1-x y}\right)
$$

The general equation We obtain similar finite identities in the general ideal $n$-gon case. In general we note that equation (6) will have $(n-3)$ independent variables and will be given by the summation of evaluating $\mathcal{L}$ on $\frac{n(n-3)}{2}$ rational functions in the $(n-3)$ variables.

\subsection{The regular ideal $n$-gon relation}

We now consider the dilogarithm equation for the specific case of a regular ideal n-gon. In this case the cross ratios can be calculated and the dilogarithm formulas for specific values of the dilogarithm function.

We consider a regular ideal $n$-gon in with center 0 in the Poincaré disk model and vertices at $v_{k}=u^{k}, k=0, \ldots, n-1$ for $u=e^{\frac{2 \pi i}{n}}$. Then equation (6) can be thought of as an equation on the roots of the polynomial $z^{n}=1$. We have

$$
\left[v_{0}, v_{1}, v_{r}, v_{r+1}\right]=-\frac{(1-u)\left(u^{r+1}-u^{r}\right)}{\left(1-u^{r}\right)\left(u^{r+1}-u\right)}=\frac{u^{r}(u-1)^{2}}{u \cdot\left(u^{r}-1\right)^{2}}=\frac{\sin ^{2}\left(\frac{\pi}{n}\right)}{\sin ^{2}\left(\frac{r \pi}{n}\right)} .
$$

For $r<n / 2$ there are exactly $n$ distinct perpendiculars between sides separated by $r$ sides and for $r=n / 2$ there are $n / 2$ such sides. To take care of the even and odd case simultaneously we let $e_{n}$ be 1 if $n$ is even and 0 if $n$ is odd. Therefore we have

$$
\sum_{r=2}^{\lceil n / 2\rceil-1} n \cdot \mathcal{L}\left(\frac{\sin ^{2}\left(\frac{\pi}{n}\right)}{\sin ^{2}\left(\frac{r \pi}{n}\right)}\right)+e_{n} \frac{n}{2} \cdot \mathcal{L}\left(\sin ^{2}\left(\frac{\pi}{n}\right)\right)=\frac{(n-3) \pi^{2}}{6}
$$

where $\lceil x\rceil$ is the least integer greater than or equal to $x$. Dividing by $n$ we get

$$
\sum_{r=2}^{\lceil n / 2\rceil-1} \mathcal{L}\left(\frac{\sin ^{2}\left(\frac{\pi}{n}\right)}{\sin ^{2}\left(\frac{r \pi}{n}\right)}\right)+\frac{e_{n}}{2} \cdot \mathcal{L}\left(\sin ^{2} \frac{\pi}{n}\right)=\frac{(n-3) \pi^{2}}{6 n} .
$$


Limiting case We let $n$ go to infinity and obtain the equation

$$
\lim _{n \rightarrow \infty}\left(\sum_{r=2}^{\lceil n / 2\rceil-1} \mathcal{L}\left(\frac{\sin ^{2}\left(\frac{\pi}{n}\right)}{\sin ^{2}\left(\frac{r \pi}{n}\right)}\right)+\frac{e_{n}}{2} \cdot \mathcal{L}\left(\sin ^{2} \frac{\pi}{n}\right)\right)=\lim _{n \rightarrow \infty} \frac{(n-3) \pi^{2}}{6 n}=\frac{\pi^{2}}{6}
$$

This gives a Rogers $\mathcal{L}$-function series relation due to Lewin [9, page 298]

$$
\sum_{r=2}^{\infty} \mathcal{L}\left(\frac{1}{r^{2}}\right)=\frac{\pi^{2}}{6}
$$

Regular ideal quadrilateral This case is trivial: $a_{1}=a_{2}=-1, b_{1}=b_{2}=1 / 2$ and equations (8) and (9) give the classical evaluations

$$
\mathcal{L}(-1)=-\frac{\pi^{2}}{12} \quad \text { and } \quad \mathcal{L}\left(\frac{1}{2}\right)=\frac{\pi^{2}}{12} .
$$

Regular ideal pentagon, Golden Mean For the regular ideal pentagon, the orthospectrum consists of 5 geodesics each of the same length $l$. Using the formula above for $n=5, r=2$ we obtain that $l$ satisfies

$$
\cosh ^{2}\left(\frac{l}{2}\right)=\frac{2}{\sqrt{5}+3}=\phi^{2}
$$

where $\phi$ is the golden mean. Therefore as $\phi^{2}=\phi+1$

$$
\sinh ^{2}\left(\frac{l}{2}\right)=\phi^{2}-1=\phi
$$

and we have $a=-\phi^{-1}$. Thus the Length Spectrum Identity gives the classical relations of Landen:

$$
\mathcal{L}\left(-\phi^{-1}\right)=-\frac{\pi^{2}}{15} \quad \mathcal{L}\left(\phi^{-2}\right)=\frac{\pi^{2}}{15}
$$

Applying the quadrilateral relations (8), (9) we also get

$$
\mathcal{L}(-\phi)=-\frac{\pi^{2}}{6}-\mathcal{L}\left(-\phi^{-1}\right)=-\frac{\pi^{2}}{10} .
$$

The regular ideal hexagon For a regular ideal hexagon, there are 9 elements of the orthospectrum, with the 6 being perpendicular to sides one apart and three being perpendicular to opposite sides. Putting $n=6$ into equation (13) above then gives

$$
6 \mathcal{L}\left(\frac{1}{3}\right)+3 \mathcal{L}\left(\frac{1}{4}\right)=\frac{\pi^{2}}{2} .
$$


Ramanujan identities We can form an elementary ideal hexagon by gluing two regular ideal quadrilaterals along a common edge. Then calculating orthospectra, the length spectrum identity gives

$$
2 \mathcal{L}\left(\frac{1}{2}\right)+2 \mathcal{L}\left(\frac{1}{3}\right)+4 \mathcal{L}\left(\frac{1}{4}\right)+\mathcal{L}\left(\frac{1}{9}\right)=\frac{\pi^{2}}{2}
$$

Therefore as $\mathcal{L}\left(\frac{1}{2}\right)=\frac{\pi^{2}}{12}$ we obtain

$$
2 \mathcal{L}\left(\frac{1}{3}\right)+4 \mathcal{L}\left(\frac{1}{4}\right)+\mathcal{L}\left(\frac{1}{9}\right)=\frac{\pi^{2}}{3}
$$

We see that taking linear combinations of equations (14) and (15), we obtain the Ramanujan identities in equations (5).

Before we prove the main theorem, we first consider the geometry of ideal quadrilaterals in the hyperbolic plane.

\section{Intersections with ideal quadrilaterals}

Given two disjoint geodesics $g_{1}, g_{2}$ with perpendicular distance $l$ between them, let $Q$ be the ideal quadrilateral with opposite sides $g_{1}, g_{2}$. Then we can map $Q$ by a Móbius transformation to the ideal quadrilateral $Q_{a}$ in the upper half-plane with vertices $a, 0,1, \infty \in \overline{\mathbb{R}}$ where $a<0$. Similarly we can map $Q$ to the ideal quadrilateral $Q_{b}$ in the upper half-plane with vertices $0, b, 1, \infty \in \overline{\mathbb{R}}$ where $b>0$. Using cross-ratios we have that

$$
a=-\frac{1}{\sinh ^{2} \frac{l}{2}} \quad b=\frac{1}{\cosh ^{2} \frac{l}{2}} .
$$

The choice of normalization $Q_{a}, Q_{b}$ leads to the equivalent forms of the Length Spectrum Identity. We choose normalization $Q_{a}$ for our calculations.

If $x, y \in \mathbb{R}, x \neq y$, we let $g(x, y)$ be the geodesic in the upper half plane with end points $x, y$. Then for $(x, y) \in(a, 0) \times(1, \infty)$, the geodesic $g(x, y)$ intersects $Q_{a}$ in a definite length denoted $L_{a}(x, y)$. Similarly for $(x, y) \in(0, b) \times(1, \infty)$, the geodesic $g(x, y)$ intersects $Q_{b}$ in a definite length denoted $L_{b}(x, y)$.

Lemma 6.1 If $k=a$ or $b$, the map $L_{k}$ is given

$$
L_{k}(x, y)=\frac{1}{2} \ln \left(\frac{y(y-k)(x-1)}{x(x-k)(y-1)}\right)=\frac{1}{2} \ln \frac{f_{k}(y)}{f_{k}(x)} .
$$




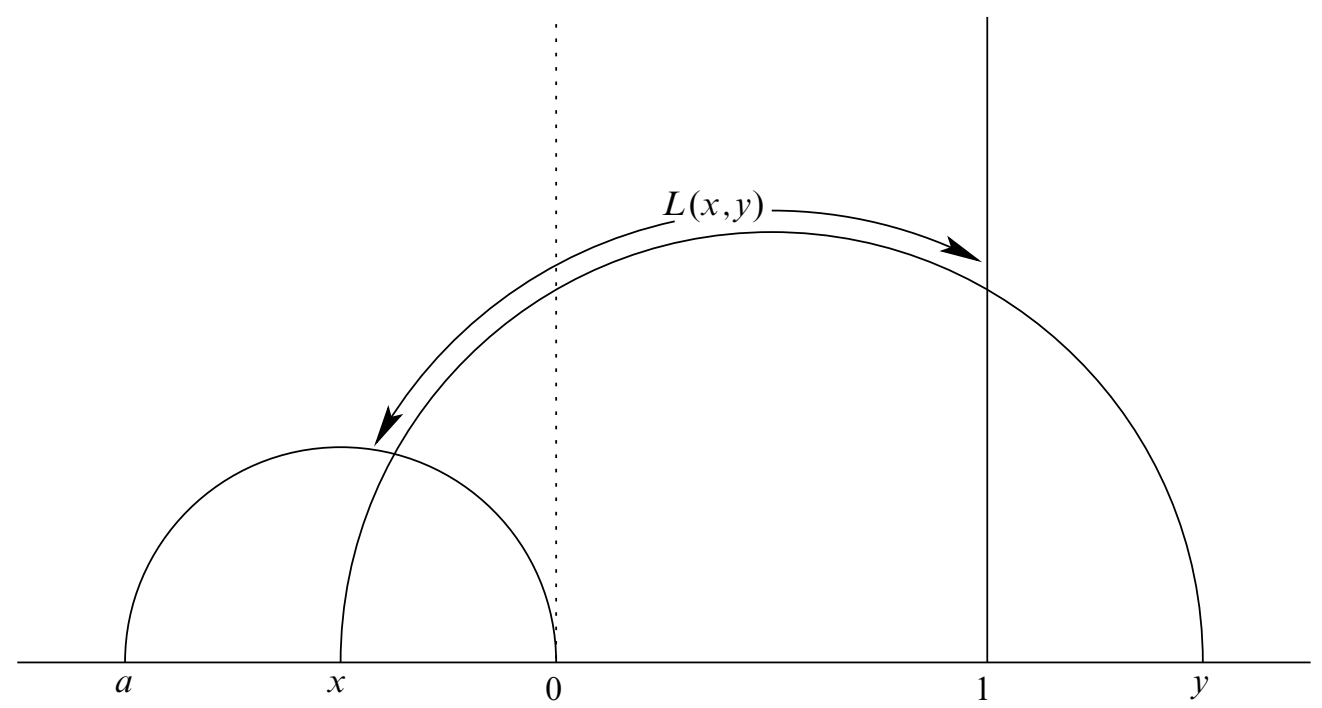

Figure 1: Length intersection function $L_{a}(x, y)$

where

$$
f_{k}(x)=\frac{x(x-k)}{x-1}
$$

Proof Let $T$ be the ideal triangle with vertices $0,1, \infty$. Let $l_{1}:(-\infty, 0) \times(0,1) \rightarrow \mathbb{R}$ and $l_{2}:(-\infty, 0) \times(1, \infty) \rightarrow \mathbb{R}$ be given by letting $l_{1}(x, y)$ be the length of the intersection of $g(x, y)$ with $T$ and $l_{2}(x, y)$ be the length of the intersection of $g(x, y)$ with $T$. By a previous paper of the author and Dumas [3], the functions $l_{i}$ are given by

$$
l_{1}(x, y)=\frac{1}{2} \ln \left(\frac{1-x}{1-y}\right) \quad l_{2}(x, y)=\frac{1}{2} \ln \left(\frac{y(x-1)}{x(y-1)}\right) .
$$

To calculate $L_{a}$, we split the quadrilateral $Q_{a}$ by the vertical line at $x=0$ into two ideal triangles $T_{1}, T_{2}$ where $T_{1}$ has vertices $0,1, \infty$ and $T_{2}$ has vertices $a, 0, \infty$. Then $T_{1}=T$ and $f_{2}(z)=z / a$ sends $T_{2}$ to $T$. Therefore

$$
L_{a}(x, y)=l_{2}(x, y)+l_{1}(y / a, x / a)
$$

Therefore

$$
\begin{aligned}
L_{a}(x, y)=\frac{1}{2} \ln \left(\frac{y(x-1)}{x(y-1)}\right)+ & \frac{1}{2} \ln \left(\frac{1-y / a}{1-x / a}\right)= \\
& \frac{1}{2} \ln \left(\frac{y(x-1)(a-y)}{x(y-1)(a-x)}\right)=\frac{1}{2} \ln \left(\frac{y(y-a)(x-1)}{x(x-a)(y-1)}\right) .
\end{aligned}
$$


To calculate $L_{b}$ we note that the map

$$
g(z)=\frac{z-b}{1-b}
$$

sends $Q_{b}$ to $Q_{a}$ where $a=g(0)=\frac{-b}{1-b}$. Therefore

$$
L_{b}(x, y)=L_{a}(g(x), g(y))=\frac{1}{2} \ln \left(\frac{\frac{y-b}{1-b}\left(\frac{y-b}{1-b}-\frac{-b}{1-b}\right)\left(\frac{x-b}{1-b}-1\right)}{\frac{x-b}{1-b}\left(\frac{x-b}{1-b}-\frac{-b}{1-b}\right)\left(\frac{y-b}{1-b}-1\right)}\right) .
$$

Simplifying we get

$$
L_{b}(x, y)=\frac{1}{2} \ln \left(\frac{y(y-b)(x-1)}{x(x-b)(y-1)}\right)
$$

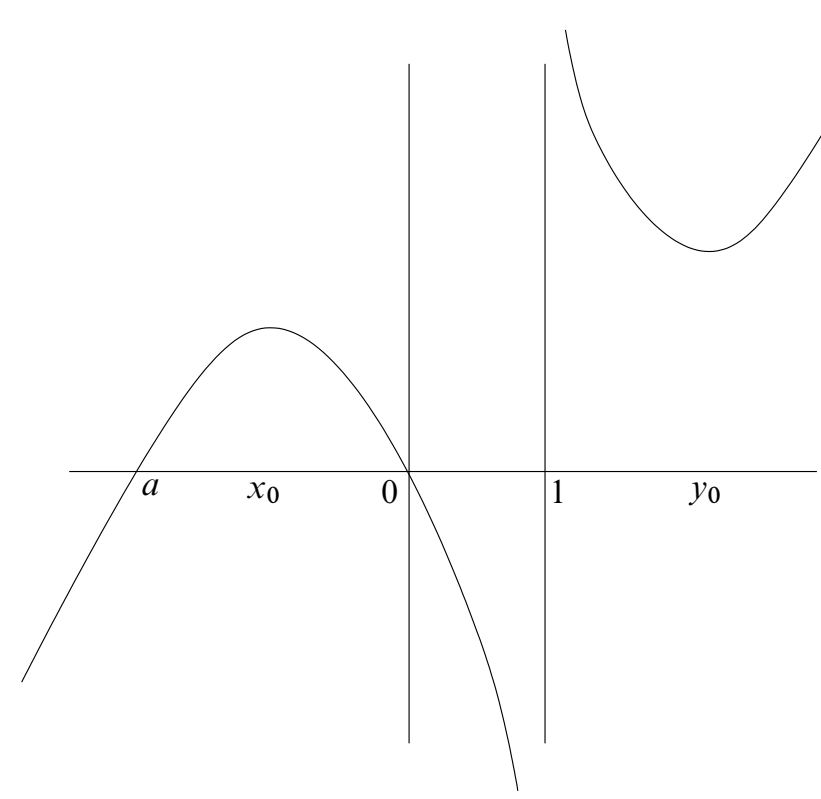

Figure 2: Graph of function $f_{a}(x)$

We consider the rational function $f_{k}(x)$ defined above. Differentiating we have

$$
f_{k}^{\prime}(x)=\frac{(2 x-k)(x-1)-1 .\left(x^{2}-k x\right)}{(x-1)^{2}}=\frac{x^{2}-2 x+k}{(x-1)^{2}} .
$$

Therefore $f_{k}(x)$ has two critical points $1 \pm \sqrt{1-k}$. We label the critical points $x_{0}=1-\sqrt{1-k}$ and $y_{0}=1+\sqrt{1-k}$. 


\section{Proof of the summation identity}

By definition

$$
\left(L_{*} \Omega\right)(\phi)=\int_{T_{1}(S)} \phi(L(v)) d \Omega .
$$

Let $\alpha, \beta$ be two arcs in $S_{\lambda}$ with endpoints on $\partial S_{\lambda}$. Then we say $\alpha \sim \beta$ if they are homotopic relative to the boundary $\partial S_{\lambda}$.

We define the sets $A_{i}=\left\{v \in T_{1}(S) \mid \alpha(v) \sim \alpha_{i}\right\}$. Also for each $\lambda$-cusp $c$ we define $A_{c}=\left\{v \in T_{1}(S) \mid \alpha(v) \sim c\right\}$ where $\alpha(v) \sim c$ if $\alpha(v)$ can be homotoped (rel boundary) out the $\operatorname{cusp} c$. Note that for $v \in A_{i}$ or $v \in A_{c}, L(v)$ is finite. Finally we define the set $A_{\infty}$ to be all $v$ not in any $A_{i}$ or $A_{c}$. By definition, the sets $A_{i}, A_{c}, A_{\infty}$ form a partition of $T_{1}(S)$. If we double $S_{\lambda}$ along its boundary, the geodesic $\operatorname{arcs} \alpha_{i}$ correspond to a subset of the geodesics of the doubled surface. Therefore as the length spectrum of the doubled surface is countable, so is the collection of $\operatorname{arcs} \alpha_{i}$ in $S_{\lambda}$. Also, by ergodicity of geodesic flow on $S$ (see Hopf [8]), the set $A_{\infty}$ is a measure zero.

Therefore

$$
\left(L_{*} \Omega\right)(\phi)=\sum_{i} \int_{A_{i}} \phi(L(v)) d \Omega+\sum_{c} \int_{A_{c}} \phi(L(v)) d \Omega .
$$

We let

$$
a_{i}=-\frac{1}{\sinh ^{2} \frac{l_{i}}{2}} .
$$

Then setting $Q_{i}=Q_{a_{i}}$, we have that $Q_{i}$ is a quadrilateral with perpendicular of length $l_{i}$. We lift $\alpha_{i}$ to the upper half plane so that it is the perpendicular of length $l_{i}$ in $Q_{i}$. We lift each $\lambda$-cusp $c$ to the ideal vertex at infinity between the vertical geodesics $x=0, x=1$. Let $T$ be the ideal triangle with vertices $0,1, \infty \in \overline{\mathbb{R}}$.

If $v \in T_{1}\left(\mathbb{H}^{2}\right)$ in the upper half plane, we define $g(v)$ to be the geodesic with tangent vector $v$. We also denote the endpoints of $g(v)$ by $(x(v), y(v))$.

We lift the set $A_{i}$ to the set $A_{i}^{\prime} \subseteq T_{1}\left(Q_{i}\right)$. Then for $v \in A_{i}^{\prime}$ the geodesic $\operatorname{arc} \alpha^{\prime}(v)=$ $Q_{i} \cap g(v)$ is a lift of $\alpha(v)$. Similarly we lift $A_{c}$ to the set $A_{c}^{\prime} \subseteq T_{1}(T)$.Then for $v \in A_{c}^{\prime}$ the geodesic arc $\alpha^{\prime}(v)=T \cap g(v)$ is a lift of $\alpha(v)$. By abuse of notation we also let $\Omega$ be the volume measure on $T_{1}\left(\mathbb{H}^{2}\right)$. We parameterize $T_{1}\left(\mathbb{H}^{2}\right)$ by $(x, y, l) \in \overline{\mathbb{R}} \times \overline{\mathbb{R}} \times \mathbb{R}$ where $(x, y, l)$ corresponds to the vector $v$ such that $g(v)$ has ordered endpoints $(x, y)$ and $v$ has basepoint on $g(v)$ a distance $l$ from the highest point of $g(v)$ in the upper half-plane. Then the volume form $\Omega$ can be written as (see Nicholls [10])

$$
d \Omega=\frac{2 d x d y d l}{(x-y)^{2}} .
$$


Therefore

$$
\int_{A_{c}} \phi(L(v)) d \Omega=\int_{A_{c}^{\prime}} \frac{2 \cdot \phi(L(v)) d x d y d l}{(x-y)^{2}} .
$$

We note that $L(v)$ only depends on the endpoints and therefore we can write $L(v)=$ $L(x, y)$. If $v \in A_{c}^{\prime}$ then either $(x, y)$ or $(y, x) \in(-\infty, 0) \times(1, \infty)$. Integrating over $l$ we have

$$
\int_{A_{c}^{\prime}} \frac{2 \cdot \phi(L(v)) d x d y d l}{(x-y)^{2}}=\int_{-\infty}^{0} \int_{1}^{\infty} \frac{4 \cdot \phi(L(x, y)) L(x, y) d x d y}{(x-y)^{2}} .
$$

By a previous paper of the author and Dumas [3],

$$
\int_{-\infty}^{0} \int_{1}^{\infty} \frac{4 \cdot \phi(L(x, y)) L(x, y) d x d y}{(x-y)^{2}}=\int_{0}^{\infty} \frac{4 \cdot \phi(L) \cdot L^{2} d L}{\sinh ^{2} L} .
$$

Thus as there are $N_{\lambda} \lambda$-cusps we have

$$
\sum_{c} \int_{A_{c}} \phi(L(v)) d \Omega=N_{\lambda} \cdot \int_{0}^{\infty} \frac{4 \cdot \phi(L) \cdot L^{2} d L}{\sinh ^{2} L}=M_{\infty}(\phi)
$$

where $M_{\infty}$ is the measure with infinitesimal

$$
d M_{\infty}=\frac{4 N_{\lambda} x^{2} d x}{\sinh ^{2} x} .
$$

Similarly we have by lifting $A_{i}$ to $A_{i}^{\prime}$ that

$$
\int_{A_{i}} \phi(L(v)) d \Omega=\int_{A_{i}^{\prime}} \frac{2 \cdot \phi(L(v)) d x d y d l}{(x-y)^{2}} .
$$

If $v \in A_{i}^{\prime}$ then either $(x, y)$ or $(y, x) \in\left(a_{i}, 0\right) \times(1, \infty)$. Integrating over $l$ we have

$$
\int_{A_{i}^{\prime}} \frac{2 \cdot \phi(L(v)) d x d y d l}{(x-y)^{2}}=\int_{a_{i}}^{0} \int_{1}^{\infty} \frac{4 \cdot \phi(L(x, y)) L(x, y) d x d y}{(x-y)^{2}} .
$$

For $a<0$ we define $M_{a}(\phi)$ to be the righthandside of the above equation. Then

$$
M_{a}(\phi)=\int_{a}^{0} \int_{1}^{\infty} \frac{4 \cdot \phi(L(x, y)) L(x, y) d x d y}{(x-y)^{2}}
$$

Then

$$
M_{\lambda}=M_{\infty}+\sum_{i} M_{a_{i}}
$$


As $M_{\lambda}=L_{*} \Omega$ it has total mass equal to the volume of $T_{1}(S)$ which is $4 \pi^{2}|\chi(S)|$. Therefore

$$
\Omega\left(T_{1}(S)\right)=4 \pi^{2}|\chi(S)|=M_{\lambda}(1)=M_{\infty}(1)+\sum_{i} M_{a_{i}}(1) .
$$

By an elementary calculation (see [3])

$$
\int_{0}^{\infty} \frac{x^{2} d x}{\sinh ^{2} x}=\frac{\pi^{2}}{6}
$$

Therefore

$$
M_{\infty}(1)=\int_{0}^{\infty} \frac{4 N_{\lambda} x^{2} d x}{\sinh ^{2} x}=4 N_{\lambda} \int_{0}^{\infty} \frac{x^{2} d x}{\sinh ^{2} x}=4 N_{\lambda} \cdot \frac{\pi^{2}}{6}=\frac{2 N_{\lambda} \pi^{2}}{3} .
$$

Using Lemma 6.1 we substitute the formula for $L_{a}(x, y)$ to obtain

$$
M_{a}(1)=\int_{a}^{0} \int_{1}^{\infty} \frac{2 \cdot \log \left(\frac{y(y-a)(x-1)}{x(x-a)(y-1)}\right) d x d y}{(x-y)^{2}}
$$

We let $F(l)=M_{a}(1)$, then by equation (17) above we obtain

$$
4 \pi^{2}|\chi(S)|=M_{\infty}(1)+\sum_{i} M_{a_{i}}(1)=\frac{2 N_{\lambda} \pi^{2}}{3}+\sum_{i} F\left(l_{i}\right)
$$

giving the summation identity

$$
\sum_{i} F\left(l_{i}\right)=4 \pi^{2}|\chi(S)|-\frac{2 N_{\lambda} \pi^{2}}{3}=\frac{2 \pi^{2}}{3}\left(6|\chi(S)|-N_{\lambda}\right)
$$

as required.

We note that it follows from the above that for

$$
a=-\frac{1}{\sinh ^{2} \frac{l}{2}} \quad b=\frac{1}{\cosh ^{2} \frac{l}{2}}
$$

then by the formulae for $L_{a}(x, y)$ and $L_{b}(x, y)$ (see Lemma 6.1) that

$$
\begin{aligned}
F(l) & =-\int_{0}^{a} \int_{1}^{\infty} \frac{2 \cdot \log \left(\frac{y(y-a)(x-1)}{x(x-a)(y-1)}\right) d x d y}{(x-y)^{2}} \\
& =\int_{0}^{b} \int_{1}^{\infty} \frac{2 \cdot \log \left(\frac{y(y-b)(x-1)}{x(x-b)(y-1)}\right) d x d y}{(x-y)^{2}}
\end{aligned}
$$




\section{Integral calculation}

In this section we find a formula for $F(l)$ by calculating an integral. We note that by the previous section, we already know that the function $F$ satisfies the functional equation (18). We will make use of this to reduce $F$ to the form we wish independent of using any classical dilogarithm relations.

Lemma 8.1 For $t<1$,

$$
\mathcal{L}(t)=\frac{1}{4} \int_{0}^{t} \int_{1}^{\infty} \frac{\log \left|\frac{y(y-t)(x-1)}{x(x-t)(y-1)}\right| d x d y}{(x-y)^{2}} .
$$

Proof We fix $t<1$ and let

$$
G(t)=\int_{0}^{t} \int_{1}^{\infty} \frac{\log \left|\frac{y(y-t)(x-1)}{x(x-t)(y-1)}\right| d x d y}{(x-y)^{2}} .
$$

Integrating by parts we get

$$
\int \frac{\log \left|\frac{y(y-t)(x-1)}{x(x-t)(y-1)}\right| d x}{(x-y)^{2}}=-\frac{\log \left|\frac{y(y-t)(x-1)}{x(x-t)(y-1)}\right|}{x-y}+\int \frac{1}{x-y}\left(\frac{1}{x-1}-\frac{1}{x}-\frac{1}{x-t}\right) d x .
$$

Using

$$
\int \frac{1}{(x-a)(x-b)} d x=\frac{1}{a-b}(\log |x-a|-\log |x-b|)
$$

we get

$$
\begin{aligned}
\int \frac{\log \left|\frac{y(y-t)(x-1)}{x(x-t)(y-1)}\right| d x}{(x-y)^{2}}= & -\frac{\log \left|\frac{y(y-t)(x-1)}{x(x-t)(y-1)}\right|}{x-y}+\frac{1}{y-1}(\log |x-y|-\log |x-1|) \\
& -\frac{1}{y}(\log |x-y|-\log |x|)-\frac{1}{y-t}(\log |x-y|-\log |x-t|) \\
= & \frac{\log \left|\frac{y(y-t)(x-1)}{x(x-t)(y-1)}\right|}{y-x}-\frac{\log |x-1|}{y-1}+\frac{\log |x|}{y}+\frac{\log |x-t|}{y-t} \\
& +\log |x-y|)\left(\frac{1}{y-1}-\frac{1}{y}-\frac{1}{y-t}\right) .
\end{aligned}
$$

We define

$$
I(y)=\int_{0}^{t} \frac{\log \left|\frac{y(y-t)(x-1)}{x(x-t)(y-1)}\right| d x}{(x-y)^{2}} .
$$


To evaluate the improper integral $I(y)$ we gather the divergent terms to find their limits. Therefore

$$
\begin{aligned}
I(y)= & \lim _{x \rightarrow 0} \log |x|\left(\frac{1}{y-x}-\frac{1}{y}\right)+\lim _{x \rightarrow t} \log |x-t|\left(\frac{1}{y-t}-\frac{1}{y-x}\right) \\
& -\frac{\log \left|\frac{y(y-t)}{t(y-1)}\right|}{y}-\frac{\log |t|}{y-t}-\log |y|\left(\frac{1}{y-1}-\frac{1}{y}-\frac{1}{y-t}\right)+\frac{\log \left|\frac{y(y-t)(t-1)}{t(y-1)}\right|}{y-t} \\
& -\frac{\log |t-1|}{y-1}+\frac{\log |t|}{y}+\log |t-y|\left(\frac{1}{y-1}-\frac{1}{y}-\frac{1}{y-t}\right) .
\end{aligned}
$$

By elementary calculus, both limits are zero. As $y>1$ and $t<1$, when we gather the remaining terms by common denominators and get

$$
\begin{aligned}
I(y)=\frac{2 \log |t|-2 \log (y-t)+\log (y-1)}{y} & +\frac{\log (y-t)-\log (1-t)-\log (y)}{y-1} \\
& +\frac{\log (1-t)+2 \log (y)-2 \log |t|-\log (y-1)}{y-t} .
\end{aligned}
$$

We now rewrite in the following form:

$$
\begin{aligned}
I(y) & =\left(\frac{\log (y-1)}{y}-\frac{\log (y)}{y-1}\right)+2\left(\frac{\log \left|\frac{y}{t}\right|}{y-t}-\frac{\log \left|\frac{y-t}{t}\right|}{y}\right)+\left(\frac{\log \left(\frac{y-t}{1-t}\right)}{y-1}-\frac{\log \left(\frac{y-1}{1-t}\right)}{y-t}\right) \\
& =I_{1}(y)+I_{2}(y)+I_{3}(y)
\end{aligned}
$$

Before we calculate the integral of $I(y)$ we note some properties of the dilogarithm. As the dilogarithm function $\mathrm{Li}_{2}$ satisfies

$$
\operatorname{Li}_{2}(z)=-\int_{0}^{z} \frac{\log (1-t)}{t} d t .
$$

Then for $x<1$ we have that $\mathcal{L}$ has derivative

$$
\begin{aligned}
\mathcal{L}^{\prime}(x) & =\frac{d}{d x}\left(\operatorname{Li}_{2}(x)+\frac{1}{2} \log |x| \log (1-x)\right) \\
& =-\frac{\log (1-x)}{x}+\frac{1}{2}\left(\frac{\log (1-x)}{x}-\frac{\log |x|}{1-x}\right) \\
& =-\frac{1}{2}\left(\frac{\log (1-x)}{x}+\frac{\log |x|}{1-x}\right) .
\end{aligned}
$$

We let

$$
J(y)=2 \mathcal{L}(1-y)-4 \mathcal{L}\left(\frac{t}{y}\right)-2 \mathcal{L}\left(\frac{1-y}{1-t}\right)=J_{1}(y)+J_{2}(y)+J_{3}(y) .
$$


Then differentiating we get

$$
\begin{aligned}
J_{1}^{\prime}(y) & =2 \cdot\left(-\frac{1}{2}\right) \cdot(-1) \cdot\left(\frac{\log (1-(1-y)}{1-y}+\frac{\log |1-y|}{1-(1-y)}\right) \\
& =\left(\frac{\log (y-1)}{y}-\frac{\log (y)}{y-1}\right)=I_{1}(y) . \\
J_{3}^{\prime}(y) & =-2 \cdot\left(-\frac{1}{2}\right) \cdot\left(-\frac{1}{1-t}\right) \cdot\left(\frac{\log \left(1-\frac{1-y}{1-t}\right)}{\frac{1-y}{1-t}}+\frac{\log \left|\frac{1-y}{1-t}\right|}{1-\frac{1-y}{1-t}}\right) \\
& =\left(\frac{\log \left(\frac{y-t}{1-t}\right)}{y-1}-\frac{\log \left(\frac{y-1}{1-t}\right)}{y-t}\right)=I_{3}(y) . \\
J_{2}^{\prime}(y) & =-4 \cdot\left(-\frac{1}{2}\right) \cdot\left(-\frac{t}{y^{2}}\right) \cdot\left(\frac{\log \left(1-\frac{t}{y}\right)}{\frac{t}{y}}+\frac{\log \left|\frac{t}{y}\right|}{1-\frac{t}{y}}\right) \\
& =-2\left(\frac{\log \left(\frac{y-t}{y}\right)}{y}+\frac{t \cdot \log \left(\frac{|t|}{y}\right)}{y(y-t)}\right) .
\end{aligned}
$$

As

we have

$$
\frac{t}{y(y-t)}=\frac{1}{y-t}-\frac{1}{y}
$$

$$
\begin{aligned}
J_{2}^{\prime}(y) & =-2\left(\frac{\log \left(\frac{y-t}{y}\right)-\log \left(\frac{|t|}{y}\right)}{y}+\frac{\log \left(\frac{|t|}{y}\right)}{y-t}\right) \\
& =-2\left(\frac{\log \left(\frac{y-t}{|t|}\right)}{y}-\frac{\log \left(\frac{y}{|t|}\right)}{y-t}\right)=I_{2}(y) .
\end{aligned}
$$

Then we have $J^{\prime}(y)=I(y)$ and therefore we have an antiderivative for $I$. Integrating we get

$$
G(t)=\int_{1}^{\infty} I(y) d y=\left.J(y)\right|_{1} ^{\infty}=\lim _{y \rightarrow \infty} J(y)-\lim _{y \rightarrow 1^{+}} J(y) .
$$

We let $\mathcal{L}_{\infty}$ be the limit if $\mathcal{L}(x)$ as $x$ tends to $-\infty$. Then

$$
\begin{aligned}
\lim _{y \rightarrow 1^{+}} J(y) & =2 \mathcal{L}(0)-4 \mathcal{L}(t)-2 \mathcal{L}(0)=4 \mathcal{L}(t) \\
\lim _{y \rightarrow \infty} J(y) & =-4 \mathcal{L}(0) .
\end{aligned}
$$

As $\mathcal{L}(0)=0$ we have

$$
G(t)=4 \mathcal{L}(t)-4 \mathcal{L}(0)=4 \mathcal{L}(t) .
$$


Therefore using equation (19) we have

$$
F(l)=-8 \mathcal{L}(a)=8 \mathcal{L}(b) .
$$

\section{Volume interpretation of $\mathcal{L}$}

Let $g_{1}, g_{2}$ be disjoint geodesics in $\mathbb{H}^{2}$ with perpendicular distance $l$ and endpoints $x_{1}, y_{1}$ and $x_{2}, y_{2}$ respectively on $\mathbb{S}^{1}$. Given $v \in T_{1}(S)$ let $g_{v}$ be the associated oriented geodesic with tangent $v$. Then we define the set

$$
C\left(g_{1}, g_{2}\right)=\left\{v \in T_{1}(S) \mid g_{v} \cap g_{1} \neq \varnothing, g_{v} \cap g_{2} \neq \varnothing\right\} .
$$

Let $t=\left[x_{1}, y_{1}, x_{2}, y_{2}\right]$, then depending on the ordering of the points on the circle we have

$$
t=\left[-1,1, e^{l},-e^{l}\right]=\frac{1}{\cosh ^{2}(l / 2)} \quad \text { or } \quad t=\left[-1,1,-e^{l}, e^{l}\right]=-\frac{1}{\sinh ^{2}(l / 2)} .
$$

It follows from the invariance of volume on $T_{1}(S)$, that the volume of $C\left(g_{1}, g_{2}\right)$ in $T_{1}(S)$ only depends on $t$. We therefore define $V(t)=\operatorname{Volume}\left(S\left(g_{1}, g_{2}\right)\right)$.

Then it follows from the main theorem that

$$
\mathcal{L}(t)= \pm \frac{1}{8} V(t)
$$

where the sign is given by the sign of $t$. Therefore we can interpret the Rogers $\mathcal{L}$-function as a signed volume function on $T_{1}(S)$ for the sets $G\left(g_{1}, g_{2}\right)$.

\section{Integral formula for $\rho$}

We let $a<0$ and

$$
L(x, y)=\frac{1}{2} \log \left(\frac{y(y-a)(x-1)}{x(x-a)(y-1)}\right)=\frac{1}{2} \log \left(\frac{f(y)}{f(x)}\right) \quad \text { for } \quad f(x)=\frac{x(x-a)}{x-1} \text {. }
$$

Taking derivatives of the length function $L(x, y)$ we have

$$
\frac{\partial L}{\partial x}=-\frac{f^{\prime}(x)}{2 f(x)} \quad \frac{\partial L}{\partial y}=\frac{f^{\prime}(y)}{2 f(y)} .
$$

By the previous section, the function $f$ has critical points $x_{0}, y_{0}$. Furthermore on $(a, 0)$ the function $f(x)$ has global maximum at $x_{0}$ and on $(1, \infty), f$ has global minimum at $y_{0}$. Therefore fixing $x$, the function $u$ : $(1, \infty) \rightarrow \mathbb{R}$ given by $u(y)=L(x, y)$ is decreasing on $\left(1, y_{0}\right)$ and increasing on $\left(y_{0}, \infty\right)$. Therefore we make the change of 
variable $t=L(x, y), x=x$. Finding inverses for $f$ we define the two functions $g_{+}$ and $g_{-}$by

$$
g_{ \pm}(x)=\frac{(a+x) \pm \sqrt{(a+x)^{2}-4 x}}{2} .
$$

Then solving $t=L(x, y)$ gives $f(y)=f(x) e^{2 t}$. Therefore on $\left(1, y_{0}\right)$ we have $y=g_{-}\left(f(x) e^{2 t}\right)$ and on $\left(y_{0}, \infty\right)$ we have $y=g_{+}\left(f(x) e^{2 t}\right)$ Therefore

$$
M_{a}(\phi)=\int_{a}^{0}\left(\int_{1}^{y_{0}}+\int_{y_{0}}^{\infty} \frac{4 . \phi(L(x, y)) L(x, y) d y}{(x-y)^{2}}\right) d x
$$

and

$$
\begin{aligned}
& \int_{1}^{y_{0}} \frac{4 \cdot \phi(L(x, y)) L(x, y) d y}{(x-y)^{2}}=\int_{\infty}^{L\left(x, y_{0}\right)} \frac{4 \cdot \phi(t) t \cdot g_{-}^{\prime}\left(f(x) e^{2 t}\right) 2 f(x) e^{2 t} d t}{\left(x-g_{-}\left(f(x) e^{2 t}\right)\right)^{2}} \\
& \int_{y_{0}}^{\infty} \frac{4 \cdot \phi(L(x, y)) L(x, y) d y}{(x-y)^{2}}=\int_{L\left(x, y_{0}\right)}^{\infty} \frac{4 \cdot \phi(t) t \cdot g_{+}^{\prime}\left(f(x) e^{2 t}\right) 2 f(x) e^{2 t} d t}{\left(x-g_{+}\left(f(x) e^{2 t}\right)^{2}\right.}
\end{aligned}
$$

Therefore combining we have

$M_{a}(\phi)=\int_{a}^{0} \int_{L\left(x, y_{0}\right)}^{\infty} 8 \cdot \phi(t) \cdot t \cdot e^{2 t} \cdot f(x)\left(\frac{g_{+}^{\prime}\left(f(x) e^{2 t}\right)}{\left(x-g_{+}\left(f(x) e^{2 t}\right)\right)^{2}}-\frac{g_{-}^{\prime}\left(f(x) e^{2 t}\right)}{\left(x-g_{-}\left(f(x) e^{2 t}\right)\right)^{2}}\right) d t d x$

We switch the order of integration. The function $L\left(x, y_{0}\right)$ is minimum at $x_{0}$ with minimum value $l=L\left(x_{0}, y_{0}\right)$ being the length of the perpendicular (see figure 2). Thus we integrate $t$ from $l$ to infinity. The integral in the $x$ direction is between the two $x$ solutions of $t=L\left(x, y_{0}\right)$ which are solutions to $f(x)=f\left(y_{0}\right) e^{-2 t}$. Thus we integrate $x$ from $g_{-}\left(f\left(y_{0}\right) e^{-2 t}\right)$ to $g_{+}\left(f\left(y_{0}\right) e^{-2 t}\right)$ giving

$$
\begin{aligned}
M_{a}(\phi)= & \int_{l_{a}}^{\infty} 8 . \phi(t) \cdot t \cdot e^{2 t} d t \\
& \left(\int_{g_{-}\left(f\left(y_{0}\right) e^{-2 t}\right)}^{g_{+}\left(f\left(y_{0}\right) e^{-2 t}\right)}\left(\frac{g_{+}^{\prime}\left(f(x) e^{2 t}\right)}{\left(x-g_{+}\left(f(x) e^{2 t}\right)\right)^{2}}-\frac{g_{-}^{\prime}\left(f(x) e^{2 t}\right)}{\left(x-g_{-}\left(f(x) e^{2 t}\right)\right)^{2}}\right) f(x) d x\right) .
\end{aligned}
$$

Therefore

$$
M_{a}(\phi)=\int_{0}^{\infty} \phi(t) \cdot \rho(l, t) d t
$$

where

$$
\begin{aligned}
& \rho(l, t)=8 t e^{2 t} \chi_{[l, \infty)} \\
&\left(\int_{g_{-}\left(f\left(y_{0}\right) e^{-2 t}\right)}^{g_{+}\left(f\left(y_{0}\right) e^{-2 t}\right)}\left(\frac{g_{+}^{\prime}\left(f(x) e^{2 t}\right)}{\left(x-g_{+}\left(f(x) e^{2 t}\right)\right)^{2}}-\frac{g_{-}^{\prime}\left(f(x) e^{2 t}\right)}{\left(x-g_{-}\left(f(x) e^{2 t}\right)\right)^{2}}\right) f(x) d x\right),
\end{aligned}
$$


and

$$
f(x)=\frac{x(x-a)}{x-1} \quad \text { where } \quad a=-\frac{1}{\sinh ^{2} l / 2} .
$$

Therefore

$$
\left(L_{*} \Omega\right)(\phi)=\int_{0}^{\infty} \phi(x) \rho(x) d x
$$

where

$$
\rho(x)=\frac{4 N_{\lambda} x^{2}}{\sinh ^{2} x}+\sum_{i} \rho\left(l_{i}, x\right)
$$

\section{Asymptotic behavior}

In this section we study the asymptotic behavior of the function $\rho(l, t)$ for large $t$.

For functions of a single variable, we write $f(x) \simeq g(x)$ as $x$ tends to $x_{0}$ if

$$
\lim _{x \rightarrow x_{0}} \frac{f(x)}{g(x)}=1 .
$$

Furthermore for functions of more than one variable, we write $f(x, y) \simeq_{x} g(x, y)$ as $x$ tends to $x_{0}$ if

$$
\lim _{x \rightarrow x_{0}} \frac{f(x, y)}{g(x, y)}=1
$$

Theorem 11.1 The measure $\rho(l, t) d x$ on the real line satisfies

$$
\lim _{t \rightarrow \infty} \frac{\rho(l, t)}{16 t^{2} e^{-2 t}}=r(l)
$$

uniformly on compact subsets of $(0, \infty)$ where

$$
r(l)=\frac{-2 a^{2}+5 a-2}{a(1-a)} \quad \text { for } \quad a=-\frac{1}{\sinh ^{2}\left(\frac{l}{2}\right)} .
$$

Proof We now show $\lim _{t \rightarrow \infty} \rho(l, t)=r(l)$ converges uniformly on compact subsets of $(0, \infty)$. Let $I \subseteq(0, \infty)$ be a compact interval. Now let $l \in I$. As before we let $a=-1 / \sinh ^{2}(l / 2)$ and define $f(x)=x(x-a) /(x-1)$ with inverses $g_{ \pm}$and critical values $x_{0}, y_{0}$. Let

$$
G(t, x)=8 t e^{2 t}\left(\frac{g_{+}^{\prime}\left(f(x) e^{2 t}\right)}{\left(x-g_{+}\left(f(x) e^{2 t}\right)\right)^{2}}-\frac{g_{-}^{\prime}\left(f(x) e^{2 t}\right)}{\left(x-g_{-}\left(f(x) e^{2 t}\right)\right)^{2}}\right) f(x) .
$$


Then for $t>l$ we have

$$
\rho(l, t)=\int_{g_{-}\left(f\left(y_{0}\right) e^{-2 t}\right)}^{g_{+}\left(f\left(y_{0}\right) e^{-2 t}\right)} G(t, x) d x .
$$

For $C>0$, we further define

$$
\rho(C, l, t)=\int_{g_{-}\left(f\left(y_{0}\right) C e^{-2 t}\right)}^{g_{+}\left(f\left(y_{0}\right) C e^{-2 t}\right)} G(t, x) d x .
$$

On the interval $[a, 0] f$ has maximum at $x_{0}$. Therefore $\rho(C, l, t)$ is defined for all $t$ such that $f\left(y_{0}\right) C e^{-2 t}<f\left(x_{0}\right)$ or

$$
t>K_{0}(C)=\frac{1}{2} \ln C+\frac{1}{2} \ln \left(\frac{f\left(y_{0}\right)}{f\left(x_{0}\right)}\right)=l+\frac{1}{2} \ln C .
$$

Considering $g_{ \pm}(x)$ for large $x$ we have

$$
g_{ \pm}(x)=\frac{(a+x) \pm \sqrt{(a+x)^{2}-4 x}}{2} \simeq \frac{(a+x)}{2}\left(1 \pm\left(1-\frac{2 x}{(a+x)^{2}}\right)\right) .
$$

Therefore

$$
g_{-}(x) \simeq \frac{(a+x)}{2}\left(1-1+\frac{2 x}{(a+x)^{2}}\right)=\frac{x}{a+x} \simeq 1-\frac{a}{x}
$$

and

$$
g_{+}(x) \simeq \frac{(a+x)}{2}\left(1+1-\frac{2 x}{(a+x)^{2}}\right)=(a+x)-\frac{x}{a+x} \simeq(a-1)+x+\frac{a}{x} .
$$

Taking leading terms we have

$$
g_{-}(x) \simeq 1, \quad g_{-}^{\prime}(x) \simeq \frac{a}{x^{2}}, \quad g_{+}(x) \simeq x, \quad g_{+}^{\prime}(x) \simeq 1 .
$$

We let

$$
I_{C}=\left[g_{-}\left(f\left(y_{0}\right) C e^{-2 t}\right), g_{+}\left(f\left(y_{0}\right) C e^{-2 t}\right)\right] .
$$

Then for $x \in I_{C}$ we have $f(x) e^{2 t} \geq C$. $f\left(y_{0}\right)$. Therefore for $C$ sufficiently large we use the above approximations to approximate $G(t, x)$ on $I_{C}$. We substitute the approximations (21) into the formula for $G(t, x)$ to define

$$
G_{1}(t, x)=8 t e^{2 t} \cdot\left(\frac{1}{\left(x-f(x) e^{2 t}\right)^{2}}-\frac{\frac{a}{\left(f(x) e^{2 t}\right)^{2}}}{(x-1)^{2}}\right) f(x) .
$$


Simplifying we have

$$
G_{1}(t, x)=8 t e^{-2 t} \cdot\left(\frac{1}{\left(1-\frac{x}{f(x) e^{2 t}}\right)^{2}}-\frac{a}{(x-1)^{2}}\right) \frac{1}{f(x)} .
$$

Noting that $f(x) e^{2 t}>C f\left(y_{0}\right)$ on $I_{C}$, then for large $C$ the quantity $\frac{x}{f(x) e^{2 t}}$ is small and we obtain the approximation

$$
G_{2}(t, x)=8 t e^{-2 t} \cdot\left(1-\frac{a}{(x-1)^{2}}\right) \frac{1}{f(x)} .
$$

Therefore given an $\epsilon>0$ we can find a $K_{1}(\epsilon)$ such that

$$
\frac{G(t, x)}{G_{2}(t, x)} \in[1-\epsilon, 1+\epsilon] \quad \text { for all } C>K_{1}(\epsilon), t>K_{0}(C), x \in I_{C} .
$$

Therefore integrating

$$
\frac{1}{\rho(C, l, t)}\left(8 t e^{-2 t} \cdot \int_{g_{-}\left(f\left(y_{0}\right) C e^{-2 t}\right)}^{g_{+}\left(f\left(y_{0}\right) C e^{-2 t}\right)}\left(1-\frac{a}{(x-1)^{2}}\right) \frac{1}{f(x)} d x\right) \in[1-\epsilon, 1+\epsilon]
$$

for $C>K_{1}(\epsilon)$ and $t>K_{0}(C)$. We fix a $K>K_{1}(\epsilon)$ and define

$$
\begin{aligned}
\rho_{K}(l, t) & =8 t e^{-2 t} \cdot\left(\int_{g_{-}\left(f\left(y_{0}\right) K e^{-2 t}\right)}^{g_{+}\left(f\left(y_{0}\right) K e^{-2 t}\right)}\left(1-\frac{a}{(x-1)^{2}}\right) \frac{1}{f(x)} d x\right) \\
& =8 t e^{-2 t} \cdot\left(\int_{g_{-}\left(f\left(y_{0}\right) K e^{-2 t}\right)}^{g_{+}\left(f\left(y_{0}\right) K e^{-2 t}\right)}\left(\frac{x-1}{x \cdot(x-a)}-\frac{a}{x(x-a)(x-1)}\right) d x\right) .
\end{aligned}
$$

Integrating we have

$$
\begin{aligned}
\int\left(\frac{x-1}{x \cdot(x-a)}-\frac{a}{x(x-a)(x-1)}\right) d x & = \\
& \left.\left(\frac{1-a}{a} \ln |x|-\frac{a}{1-a} \ln |x-1|-\frac{a^{2}-3 a+1}{a(1-a)} \ln |x-a|\right)\right) .
\end{aligned}
$$

Therefore

$\rho_{K}(l, t)=\left.8 t e^{-2 t} \cdot\left(\frac{1-a}{a} \ln |x|-\frac{a}{1-a} \ln |x-1|-\frac{a^{2}-3 a+1}{a(1-a)} \ln |x-a|\right)\right|_{g_{-}\left(f\left(y_{0}\right) K e^{-2 t}\right)} ^{g_{+}\left(f\left(y_{0}\right) K e^{-2 t}\right)}$.

For $x$ small we have

$$
g_{ \pm}(x)=\frac{(a+x) \pm \sqrt{(a+x)^{2}-4 x}}{2} \simeq \frac{(a+x)}{2}\left(1 \mp\left(1-\frac{2 x}{(a+x)^{2}}\right)\right) .
$$


Therefore

$$
g_{-}(x) \simeq(a+x)-\frac{x}{a+x} \simeq a-\frac{(1-a) x}{a}, \quad g_{+}(x) \simeq \frac{x}{a+x} \simeq \frac{x}{a} .
$$

Therefore

$$
\begin{aligned}
\rho_{K}(l, t) \simeq_{t} 8 t e^{-2 t} \cdot\left(\frac{1-a}{a} \ln \left|\frac{K f\left(y_{0}\right) e^{-2 t}}{a^{2}}\right|\right. & -\frac{a}{1-a} \ln \left|\frac{1}{a-1}\right| \\
& \left.-\frac{a^{2}-3 a+1}{a(1-a)} \ln \left|\frac{a^{2}}{(1-a) f\left(y_{0}\right) K e^{-2 t}}\right|\right) .
\end{aligned}
$$

Taking limits as we have

$$
\rho_{K}(l, t) \simeq_{t}\left(16 t^{2} e^{-2 t}\right) \cdot\left(-\frac{1-a}{a}-\frac{a^{2}-3 a+1}{a(1-a)}\right)=\left(16 t^{2} e^{-2 t}\right) \cdot \frac{-2 a^{2}+5 a-2}{a(1-a)} .
$$

Therefore given $\epsilon>0$ there exists $K_{1}(\epsilon)>0$ such that for any $C>K_{1}(\epsilon)$ both

$$
\liminf _{t \rightarrow \infty} \frac{\rho(C, l, t)}{16 t^{2} e^{-2 t} r(a)} \text { and } \limsup _{t \rightarrow \infty} \frac{\rho(C, l, t)}{16 t^{2} e^{-2 t} r(a)} \text { are in }[1-\epsilon, 1+\epsilon]
$$

where

$$
r(a)=\frac{-2 a^{2}+5 a-2}{a(1-a)}
$$

We now define

$$
\begin{aligned}
\rho_{-}(C, l, t) & =\int_{g_{-}\left(f\left(y_{0}\right) e^{-2 t}\right)}^{g_{-}\left(f\left(y_{0}\right) C e^{-2 t}\right)} G(t, x) d x \\
\text { and } \quad \rho_{+}(C, l, t) & =\int_{g_{+}\left(f\left(y_{0}\right) C e^{-2 t}\right)}^{g_{+}\left(f\left(y_{0}\right) e^{-2 t}\right)} G(t, x) d t .
\end{aligned}
$$

Then by definition

$$
\rho(l, t)=\rho(C, l, t)+\rho_{-}(C, l, t)+\rho_{+}(C, l, t) .
$$

We now bound the functions $\rho_{ \pm}(C, l, t)$. Let $I_{C}^{-}, I_{C}^{+}$be the given intervals.

On the interval $I, g_{ \pm}\left(f(x) e^{2 t}\right)>1$ and $x<0$ so $\left(x-g_{ \pm}\left(f(x) e^{2 t}\right)\right)^{2}>1$. Also as $g_{-}^{\prime}\left(f(x) e^{2 t}\right)<0$ we have

$$
\begin{aligned}
|G(t, x)| & =\left(8 t \cdot e^{2 t}\right) \cdot\left(\frac{g_{+}^{\prime}\left(f(x) e^{2 t}\right)}{\left(x-g_{+}\left(f(x) e^{2 t}\right)\right)^{2}}-\frac{g_{-}^{\prime}\left(f(x) e^{2 t}\right)}{\left(x-g_{-}\left(f(x) e^{2 t}\right)\right)^{2}}\right) f(x) \\
& \leq 8 t \cdot e^{2 t} \cdot\left(g_{+}^{\prime}\left(f(x) e^{2 t}\right)-g_{-}^{\prime}\left(f(x) e^{2 t}\right)\right) f(x) .
\end{aligned}
$$


The derivative of $g_{ \pm}(x)$ is given by

$$
g_{ \pm}^{\prime}(x)=\frac{1}{2} \pm \frac{1}{2} \frac{x+a-2}{\sqrt{(a+x)^{2}-4 x}} .
$$

Therefore

$$
g_{+}^{\prime}(x)-g_{-}^{\prime}(x)=\frac{x+a-2}{\sqrt{(a+x)^{2}-4 x}} .
$$

As $f$ has critical values $f\left(x_{0}\right)$ and $f\left(y_{0}\right)$ we have that

$$
g_{+}^{\prime}(x)-g_{-}^{\prime}(x)=\frac{x+a-2}{\sqrt{\left(x-f\left(x_{0}\right)\right)\left(x-f\left(y_{0}\right)\right)}} .
$$

We note that on $I_{C}^{ \pm}$we have $f\left(y_{0}\right)<f(x) e^{2 t}<C f\left(y_{0}\right)$ then

$$
\begin{aligned}
g_{+}^{\prime}\left(f(x) e^{2 t}\right)-g_{-}^{\prime}\left(f(x) e^{2 t}\right) & \leq \frac{C f\left(y_{0}\right)+a-2}{\sqrt{\left(f\left(y_{0}\right)-f\left(x_{0}\right)\right)\left(f(x) e^{2 t}-f\left(y_{0}\right)\right)}} \\
& \leq\left(\frac{C f\left(y_{0}\right)+a-2}{\sqrt{f\left(y_{0}\right)-f\left(x_{0}\right)}}\right) \frac{e^{-t}}{\sqrt{f(x)-f\left(y_{0}\right) e^{-2 t}}} .
\end{aligned}
$$

The function $f(x)=x(x-a) /(x-1)$ has maximum at $x_{0}$ on $(a, 0)$. Therefore for $b<f\left(x_{0}\right)$

$$
f(x)-b=\frac{\left(x-g_{-}(b)\right)\left(x-g_{+}(b)\right)}{(x-1)} .
$$

As $x \in(a, 0)$ we have

$$
f(x)-b \geq\left(x-g_{-}(b)\right)\left(g_{+}(b)-x\right) .
$$

Therefore

$$
\begin{aligned}
g_{+}^{\prime}\left(f(x) e^{2 t}\right)- & g_{-}^{\prime}\left(f(x) e^{2 t}\right) \leq \\
& \left(\frac{C f\left(y_{0}\right)+a-2}{\sqrt{f\left(y_{0}\right)-f\left(x_{0}\right)}}\right) \frac{e^{-t}}{\sqrt{\left(x-g_{-}\left(f\left(y_{0}\right) e^{-2 t}\right)\right)\left(g_{+}\left(f\left(y_{0}\right) e^{-2 t}\right)-x\right)}} .
\end{aligned}
$$

Now restricting to $I_{C}^{+}$we have $x>g_{+}\left(f\left(y_{0}\right) C e^{-2 t}\right)$. Therefore for $x \in I_{C}^{+}$,

$$
\begin{aligned}
& g_{+}^{\prime}\left(f(x) e^{2 t}\right)-g_{-}^{\prime}\left(f(x) e^{2 t}\right) \leq \\
& \quad\left(\frac{C f\left(y_{0}\right)+a-2}{\sqrt{\left(f\left(y_{0}\right)-f\left(x_{0}\right)\right)\left(g_{+}\left(f\left(y_{0}\right) C e^{-2 t}\right)-g_{-}\left(f\left(y_{0}\right) e^{-2 t}\right)\right)}}\right) \frac{e^{-t}}{\sqrt{g_{+}\left(f\left(y_{0}\right) e^{-2 t}\right)-x}} .
\end{aligned}
$$


Therefore we have

$$
\rho_{+}(C, l, t) \leq \int_{I_{C}^{+}}|G(t, x)| d x \leq D(t) 8 t e^{t} \cdot \int_{I_{C}^{+}} \frac{f(x)}{\sqrt{g_{+}\left(f\left(y_{0}\right) e^{-2 t}\right)-x}} d t
$$

where $D(t)$ is the constant

$$
D(t)=\left(\frac{C f\left(y_{0}\right)+a-2}{\sqrt{\left(f\left(y_{0}\right)-f\left(x_{0}\right)\right)\left(g_{+}\left(f\left(y_{0}\right) C e^{-2 t}\right)-g_{-}\left(f\left(y_{0}\right) e^{-2 t}\right)\right)}}\right) .
$$

As $f(x)=x(x-a) /(x-1)$ then, $0<f(x) \leq a x$ on $(a, 0)$ we have

$$
\rho_{+}(C, l, t) \leq \int_{I_{C}^{+}}|G(t, x)| d x \leq D(t) .8 \text {.a.t. } e^{t} \cdot \int_{I_{C}^{+}} \frac{x}{\sqrt{g_{+}\left(f\left(y_{0}\right) e^{-2 t}\right)-x}} d x .
$$

By integration we have

$$
\int_{a}^{b} \frac{x}{\sqrt{b-a}} d x=\frac{2}{3}(2 b+a) \sqrt{b-a}
$$

Therefore

$\rho_{+}(C, l, t) \leq 16 \cdot D(t) \cdot a \cdot t \cdot e^{t} \cdot\left(2 g_{+}\left(f\left(y_{0}\right) e^{-2 t}\right)+g_{+}\left(f\left(y_{0}\right) C e^{-2 t}\right)\right)$

$$
\sqrt{g_{+}\left(f\left(y_{0}\right) e^{-2 t}\right)-g_{+}\left(f\left(y_{0}\right) C e^{-2 t}\right)} .
$$

Now for $t$ large we have

$$
\lim _{t \rightarrow \infty} D(t)=\left(\frac{C f\left(y_{0}\right)+a-2}{\sqrt{\left(f\left(y_{0}\right)-f\left(x_{0}\right)\right) \cdot|a|}}\right)=D .
$$

We note for $x$ small $g_{+}(x) \simeq x / a$. Therefore

$$
\begin{aligned}
& \limsup _{t \rightarrow \infty} \mid \frac{\rho_{+}(C, l, t)}{t^{2} e^{-2 t} \mid \leq} \\
& \limsup _{t \rightarrow \infty} \frac{16 . \text { D.a.t.e } t .\left(\frac{2 f\left(y_{0}\right) e^{-2 t}+f\left(y_{0}\right) C e^{-2 t}}{a}\right) \sqrt{\frac{f\left(y_{0}\right) e^{-2 t}-f\left(y_{0}\right) C e^{-2 t}}{a}}}{t^{2} e^{-2 t}}
\end{aligned}
$$

$\limsup _{t \rightarrow \infty}\left|\frac{\rho_{+}(C, l, t)}{t^{2} e^{-2 t}}\right| \leq \limsup _{t \rightarrow \infty} \frac{16 \cdot D \cdot f\left(y_{0}\right)^{3 / 2}(C+2) \sqrt{C-1}}{t \cdot \sqrt{-a}}=0$.

Thus

$$
\lim _{t \rightarrow \infty} \frac{\rho_{+}(C, l, t)}{t^{2} e^{-2 t}}=0 .
$$


Similarly for $\rho_{-}(C, l, t)$ we once again have that

$$
\lim _{t \rightarrow \infty} \frac{\rho_{-}(C, l, t)}{t^{2} e^{-2 t}}=0 .
$$

Therefore given $\epsilon>0$ we can find $K(\epsilon)$ such that for $C>K(\epsilon)$ by equations (22)

$$
\begin{aligned}
\limsup _{t \rightarrow \infty} \frac{\rho(l, t)}{16 t^{2} e^{-2 t} r(a)} & =\limsup _{t \rightarrow \infty}\left(\frac{\rho_{-}(C, l, t)}{16 t^{2} e^{-2 t} r(a)}+\frac{\rho(C, l, t)}{16 t^{2} e^{-2 t} r(a)}+\frac{\rho_{+}(C, l, t)}{16 t^{2} e^{-2 t} r(a)}\right) \\
& =\limsup _{t \rightarrow \infty} \frac{\rho(C, l, t)}{16 t^{2} e^{-2 t} r(a)} \in[1-\epsilon, 1+\epsilon] .
\end{aligned}
$$

As $\epsilon$ is arbitrary we have

$$
\limsup _{t \rightarrow \infty} \frac{\rho(l, t)}{16 t^{2} e^{-2 t} r(a)}=1 .
$$

Similarly

$$
\liminf _{t \rightarrow \infty} \frac{\rho(l, t)}{16 t^{2} e^{-2 t} r(a)}=1 .
$$

\section{References}

[1] A Basmajian, The orthogonal spectrum of a hyperbolic manifold, Amer. J. Math. 115 (1993) 1139-1159 MR1246187

[2] B C Berndt, Ramanujan's Notebooks Part IV, Springer, New York (1994) MR1261634

[3] M Bridgeman, D Dumas, Distribution of intersection lengths of a random geodesic with a geodesic lamination, Ergodic Theory Dynam. Systems 27 (2007) 1055-1072 MR2342965

[4] M Bridgeman, J Kahn, Hyperbolic volume of manifolds with geodesic boundary and orthospectra, Geom. Funct. Anal. 20 (2010) 1210-1230 MR2746952

[5] D Calegari, Chimneys, leopard spots and the identities of Basmajian and Bridgeman, Algebr. Geom. Topol. 10 (2010) 1857-1863 MR2684984

[6] D Calegari, Bridgeman's orthospectrum identity, Topology Proc. 38 (2011) 173-179 MR2680162

[7] B Gordon, R J McIntosh, Algebraic dilogarithm identities, Ramanujan J. 1 (1997) 431-448 MR1608701 International Symposium on Number Theory (Madras, 1996)

[8] E Hopf, Statistik der geodätischen Linien in Mannigfaltigkeiten negativer Krümmung, Ber. Verh. Sächs. Akad. Wiss. Leipzig 91 (1939) 261-304 MR0001464

[9] L Lewin, editor, Structural properties of polylogarithms, Mathematical Surveys and Monographs 37, American Mathematical Society, Providence, RI (1991) MR1148371 
[10] P J Nicholls, The ergodic theory of discrete groups, London Mathematical Society Lecture Note Series 143, Cambridge University Press, Cambridge (1989) MR1041575

[11] L J Rogers, On function sum theorems connected with the series $\sum_{1}^{\infty} \frac{x^{n}}{n^{2}}$, Proc. London Math. Soc. 4 (1907) 169-189

Department of Mathematics, Boston College, Chestnut Hill, Ma 02167

bridgem@bc . edu

http: //www2.bc.edu/martin-bridgeman/

Proposed: Benson Farb

Seconded: Danny Calegari, David Gabai
Received: 27 December 2010

Accepted: 14 February 2011 